\title{
Widely Distributed Photonics-Based Dual-Band MIMO Radar for Harbour Surveillance
}

\author{
F. Scotti ${ }^{\circledR}$, S. Maresca, L. Lembo, G. Serafino ${ }^{\circledR}$, A. Bogoni ${ }^{\circledR}$, Member, IEEE, \\ and P. Ghelfi ${ }^{\circledR}$, Member, IEEE
}

\begin{abstract}
A new architecture for a widely distributed dual-band coherent multiple-input multiple-output (MIMO) radar system is illustrated, and its implementation and testing are reported. The system consists in a central unit where radar signals are coherently generated and detected, which serves multiple remote sensors connected over transparent WDM optical network. Every remote node operates coherently both in the S- and X-band, and is displaced over distances of several kilometers, allowing to monitor a scene under different angles of view. All the remote sensors share the same oscillator and digital signal processing unit, both located in the central office, allowing to perform centralized raw data fusion on the acquired signals. By virtue of the system coherence, the system takes advantage of the coherent MIMO processing strategy to offer a superior spatial resolution, which is even magnified by the dual-band approach.
\end{abstract}

Index Terms-Microwave photonics, MIMO radar, coherent radar, multisensor multiband data fusion.

\section{INTRODUCTION}

$\mathbf{S}$ URVEILLANCE applications require ever-increasing performance from radar systems. Since standalone radars are no longer sufficient to meet such operative requirements, the paradigm of multiple radars is often implemented in the form of multistatic radars, i.e. radars with multiple transmitters and/or receivers positioned in different places. They provide multiple points of view of the same area, allowing the detection of targets obscured due to the presence of obstacles (e.g. small boats hiding behind the shoreline or cargo ships), or with unfavorable angles of view. However, in multistatic radar systems, the remote nodes seldom cooperate with each other. These multiple radars act independently or with poor coordination, and they exchange only a limited amount of data. In fact, each node typically performs a local pre-processing (e.g., detection, tracking), and then sends the results to a central node for data fusion. On one hand, this solution avoids any issue related to the synchronization of the network nodes and reduces the amount of data to be shared. On the other hand, this simplification implies an evident performance limitation due to the fact that data fusion is done on already

Manuscript received June 15, 2020; revised July 6, 2020; accepted July 23, 2020. Date of publication July 29, 2020; date of current version August 6, 2020. This work was supported by the NATO SPS SOLE Project. (Corresponding author: F. Scotti.)

F. Scotti, A. Bogoni, and P. Ghelfi are with CNIT, 56124 Pisa, Italy (e-mail: filippo.scotti@cnit.it).

S. Maresca, L. Lembo, and G. Serafino are with Scuola Superiore Sant'Anna, 56124 Pisa, Italy.

Color versions of one or more of the figures in this letter are available online at http://ieeexplore.ieee.org.

Digital Object Identifier 10.1109/LPT.2020.3012731 elaborated plots and not directly on the acquired samples of each signal.

Recently, radar networks with a higher degree of coordination have been proposed, where different receivers can process signals coming from multiple emitters. These radar networks are usually named multiple-input multiple-output (MIMO) radars [1]. Unfortunately, coherent MIMO radars with widely separated antennas require precise signal synchronization and reliable large-bandwidth long-range signal distribution among the network nodes. Up to now, these two issues have represented the main limitation factors to the development of such systems in real operative scenarios.

In the last years, the advances of radio-over-fiber (RoF) technology provided a suitable solution for long distance radio frequency (RF) analog signal distribution. Moreover, photonics made the appearance in the world of radars with the development of the first prototype of a photonics-based radar system [2], capable of state-of-the-art performance [3] and improved functionalities [4].

Since in this kind of radar the signal generation and processing are based on photonics, which guarantees an unprecedented frequency flexibility, the signal distribution through optical fibers is the natural evolution, assuring high-quality and high-coherence, as well as large bandwidth connectivity between remote sensors [5]. Therefore, photonics can become an enabling technology for the realization of MIMO radar networks with widely distributed antennas. In fact, a distributed coherent photonic radar network can be envisioned consisting of a central photonic core, which is capable to generate and distribute frequency-agile radar signals via optical fibers to multiple antennas. Conversely, the RF echoes received by each antenna are locally converted to the optical domain and sent back to the central processing core to be jointly processed [6].

In order to investigate the capability of this technology, the authors in [7] also developed a proof of concept MIMO radar, consisting in 2 transmitters and 2 receivers spaced by few tens of meters. The proposed system was able to successfully resolve two targets closely-spaced in cross-range, thus demonstrating the superiority of the coherent MIMO approach compared with the simple non-coherent processing.

In this paper we further push the concept of a photonics-based MIMO radar network introducing three main innovative aspects: (i) the multiband operation which introduces a further improvement in the performance of a coherent MIMO radar, (ii) a largely distributed geometry with sensor 


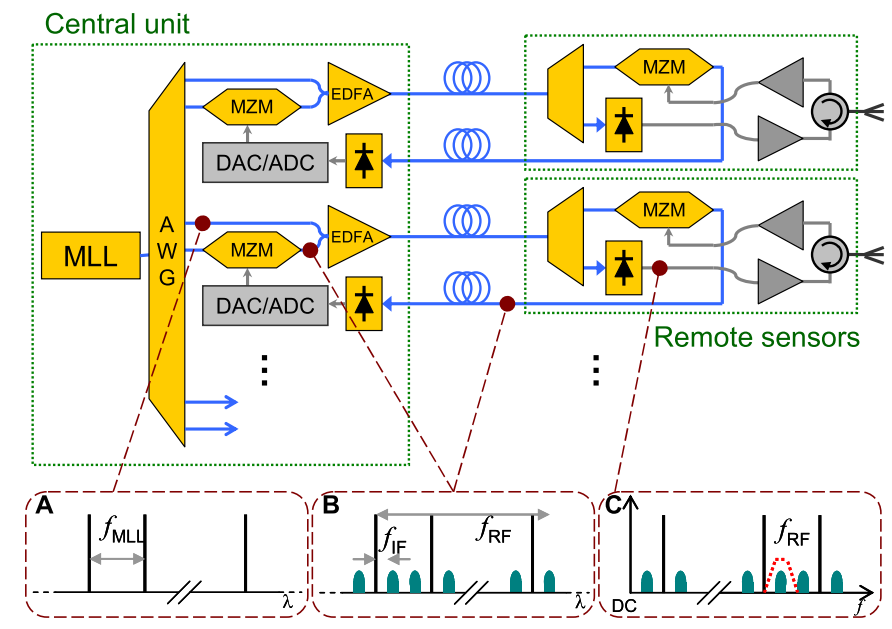

Fig. 1. Scheme of principle of the presented MIMO architecture.

spaced several km, and (iii) improved RF front-ends to allow the detection of small boats with low RCS across the whole area. In particular, we designed and developed an ad-hoc architecture for monitoring a $5 \mathrm{~km}$ wide harbour, exploiting the already existing optical network of the port for connecting the remote sensors to the central unit, and we characterized the system for verifying its suitability for the considered scenario.

\section{System ARChitecture}

The scheme of the implemented centralized architecture is reported in Fig. 1. It is composed of a central unit, hosting the photonic core as well as the intermediate frequency waveform digital generators and receivers, and multiple remote sensors (RS), hosting the opto-electric conversions and the necessary $\mathrm{RF}$ amplification stages. The central unit and the remote nodes are connected by pairs of standard optical fibers.

The photonic core is based on a mode locked laser (MLL) that generates an ultra-stable phase-locked optical frequency comb with a tone spacing $f_{\mathrm{MLL}}=400 \mathrm{MHz}$. The MLL is used as the optical local oscillator to up-convert and down-convert the radar signals to and from the different RF frequency bands, respectively, as discussed in [4]. Since the whole 6nm spectrum of the MLL is not necessary for RF frequency conversion, a 24 ports arrayed waveguide grating (AWG) divides it in multiple slices with a bandwidth of $0.4 \mathrm{~nm}$ (i.e. DWDM ITU grid), each containing over 100 laser modes (inset A), and routes them onto different output fibers. Two spectrum slices are devoted to each remote sensor. The first is intended for the radar transmitter and is amplitude-modulated in a Mach-Zehnder modulator (MZM) with the radar waveform generated at intermediate frequency $f_{\mathrm{IF}}=100 \mathrm{MHz}$, generating upper- and lower-sidebands around each laser mode (inset B). The second one is intended for the receiver, and is coupled as it is the other one, so that both signals are amplified in an erbium doped fiber amplifier (EDFA) and sent to the remote sensor. Devoting different wavelengths to each sensor on one hand improves the efficiency of the system avoiding waste of power, on the other hand allows to share the same optical fiber between different sensors or with telecom traffic, simplifying the deployment of the system. Each remote unit receives the optical signals from the central station and

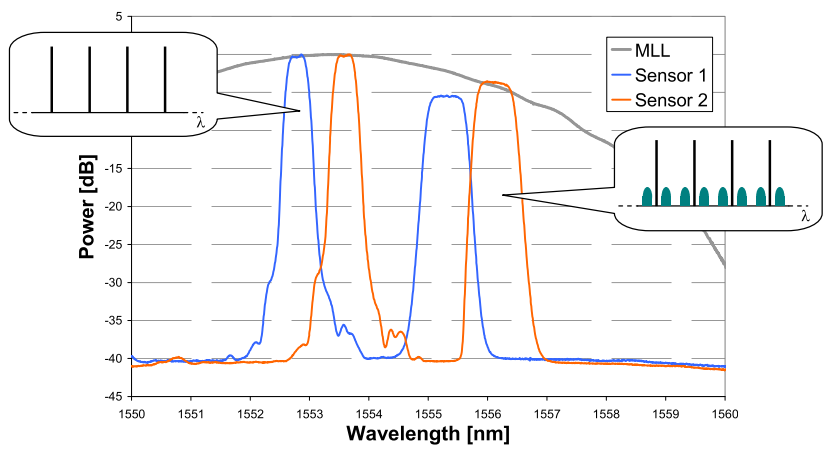

Fig. 2. Optical spectra of the MLL and of the signals feeding two different remote sensors.

separates its two optical signals with a wavelength division multiplexer (WDM). The modulated one carrying the radar waveform is received by a wideband photodiode, where all the spectral components are heterodyned together, resulting in the upconversion of the signal to every multiple frequency of the MLL (inset C) [3], [4]. Two of these replica, i.e. the one at $2.9 \mathrm{GHz}$ and the one at $9.7 \mathrm{GHz}$, are filtered and amplified up to $20 \mathrm{~W}$ in a dual-band RF front-end [4] to be transmitted.

At the receiver side, the radar echoes are amplified within the RF front-end and the resulting signals is used to modulate the second wavelength in the remote MZM. The optical signal, which now carries the radar echoes (inset B), is then sent back to the central unit via a second optical fiber. Here it is received by a low bandwidth photodiode to recover the down-converted echoes. Finally, these are low-pass filtered and then digitized in an analog to digital converter (ADC) to be digitally processed. Since both the S- and X-band signals share the same IF, a time division multiplexing approach has been implemented, so that the radar pulses in the two frequencies are transmitted alternatively.

In the current implementation two remote units have been realized. Fig. 2, shows the original optical spectrum of the MLL and its four portions feeding the units. To avoid crosstalk, transmitters and receivers use non adjacent ITU channels. Due to the $6 \mathrm{~nm}$ wide spectrum of the MLL, up to seven wavelength independent remote sensors can be implemented, with a modular approach.

\section{System Performance}

The first characterization of the developed radar system has been carried out to assess the suitability of its performance in case of widely separated sensors. To this extent, two $10 \mathrm{~km}$-long optical fiber spools have been placed between the central unit and the remote sensor, delaying the signals from and to the central unit, i.e. emulating the remotization of the sensor even beyond the envisioned application. The transmitted RF spectrum is reported in Fig. 3-A, as well as the zooms of the two bands (Fig. 3-B and -C), in case of $1 \mu$ s-long frequency chirped pulses over a pulse repetition interval (PRI) of $50 \mu \mathrm{s}$.

Moreover, in order to verify the detection capability of the system, the transmitted RF radar signal has been further delayed by RoF delay line made of a $4 \mathrm{~km}$-long optical fiber spool, opportunely attenuated, and sent back to the antenna port of the remote sensor, this emulating the radar echo 

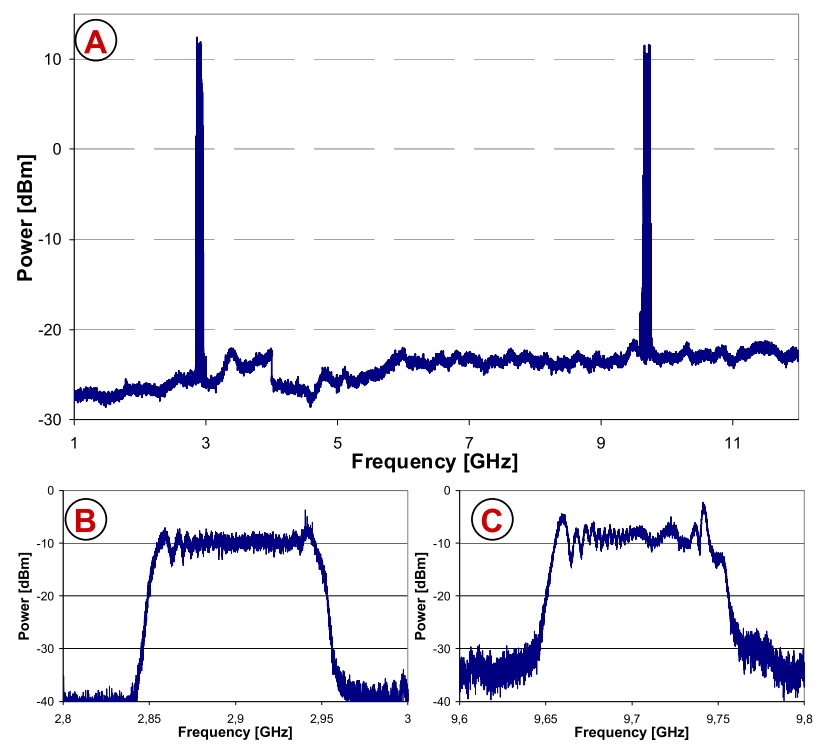

Fig. 3. A) RF spectrum of the transmitted signal from a remote sensor (RBW $3 \mathrm{MHz})$; B) Zoom of the transmitted signal in the S-Band (RBW $30 \mathrm{kHz})$; C) Zoom of the transmitted signal in the X-Band (RBW 30kHz).
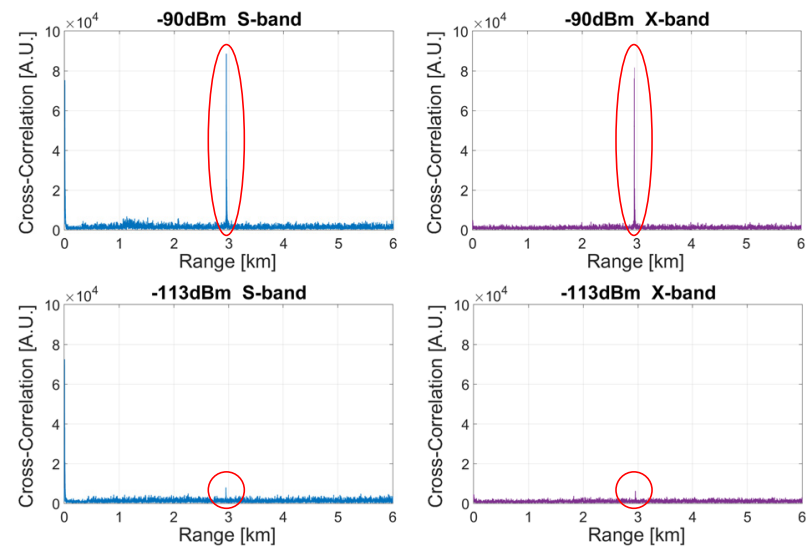

Fig. 4. Autocorrelation function for the S-band and X-band signals, with different received power levels.

from a target. Fig. 4 reports the cross-correlation between the delayed echoes and the digital reference signal for the two frequency bands and at different power levels. Considering the 1.5 refractive index of the delay line and the roundtrip time, the $4 \mathrm{~km}$ spool emulates a target $3 \mathrm{~km}$ away, as is correctly measured by the system. The noise level is almost the same in every detection, while the peak level changes. The extinction ratio between the peak and the highest point of the noise background has been measured varying the echo power level, as reported in Fig. 5. As expected, the graph shows a linear trend with the received power over $50 \mathrm{~dB}$ of dynamic, and about $3 \mathrm{~dB}$ better performance for the X-band curve with respect to the S-band. This is mainly due to the slightly better low noise amplifier employed in the X-band receiver amplification chain. Nevertheless, considering the lower atmospheric propagation losses of the S-band signals, the developed system should detect targets as small as 1 square meter radar cross section (RCS) more than $2 \mathrm{~km}$ away, such as small tenders that could be a threat for big cargo ships.

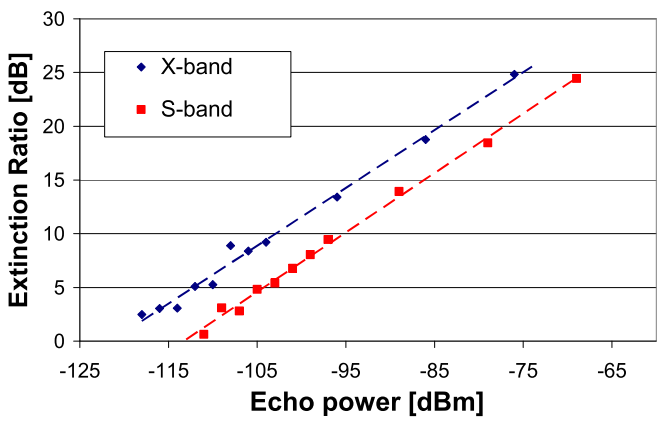

Fig. 5. Extinction ratio of the autocorrelation function peak and the background noise for the S-band and X-band signals, as a function of the received power.

\section{COHERENT MIMO}

In a MIMO radar, the target position is estimated as the location for which its log-likelihood function, conditional on the set of all the available received signals, reaches the maximum. First, the cross-correlations between the received and transmitted base-band equivalent signals are calculated. Then, the cross-correlation vectors are mapped via geometric transformation into complex 2D matrices. Here, the peaks displayed within the cross-correlation vector transform into ellipsoidal wave fronts at the varying of range and cross-range. There are two ways to calculate the MIMO ambiguity function out of these matrices, named "searching" and "imaging" modes. The first mode consists in calculating the "non-coherent" MIMO ambiguity function, whereas the second mode consists in calculating the so-called "coherent" MIMO ambiguity function. Mathematical details on MIMO processing can be found in [1] and references therein.

To exploit the "imaging" mode, the coherence between all the different sensors is a fundamental requirement. Here the coherence is guaranteed by the use of the same optical clock (i.e. the MLL) first in transmission for the up-conversion and then at the receiver for the down-conversion, for all the radar sensors as reported in Fig. 1. As demonstrated in [2], the RF signals generated by means of a MLL present a very high phase stability, with a time jitter in the order of tens of fs.

In Fig. 6-A we report the phase stability analysis measuring the back-to-back received signal (i.e. with the transmitter directly connected to the receiver) at the central unit, in the case the connection between the central and remote units is realized with a simple $2 \mathrm{~m}$ fiber pair connection, or using the two $10 \mathrm{~km}$ fiber spools, over a period of four hours. The measure shows that the long fiber spools have a negligible effect on the short period, but introduce a slight drift of about 5 deg over multiple hours. This drift, if not compensated, could in principle affect the performance of the MIMO processing, that requires a precise knowledge of the phase of the different emitters. However, as reported in [8], if the angular jitter of the architecture is lower than $6 \mathrm{deg}$, the effects on the MIMO coherent detection performance are negligible.

Before deploying the system a Back to Back experiment has been set up for demonstrating the effectiveness of the dual-band coherent MIMO. As depicted in Fig. 6-B, the two RS have been connected to the central unit via $3.6 \mathrm{~km}$ and a $4.1 \mathrm{~km}$ fiber pairs representing the actual different paths 

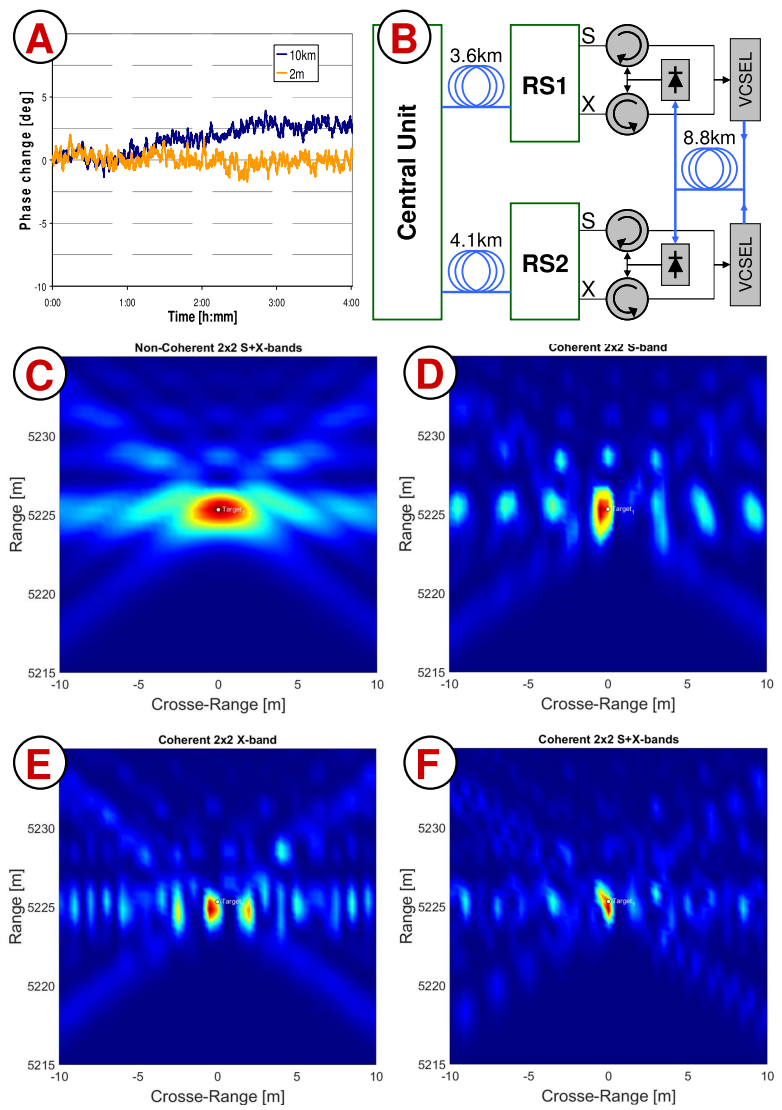

Fig. 6. A) Phase stability of the received back-to-back signal; B) Exterimental BtB setup; C) Non coherent detection; D) S-Band coherent processing; E) X-Band coherent processing; F) Dual band coherent processing.

between the sensors and the office, and each RS has been connected to a target emulator composed by a VCSEL-based wideband RoF delay line. Both RoF are coupled together sharing the same $8.8 \mathrm{~km}$ fiber spool, that for geometric considerations emulates a scenario in which both the RSs are equidistant from the target. Fig. 6 reports the target as detected with a non-coherent MIMO processing, and in case of single-band coherent MIMO both in S- and X-band, as well as the result of a dual-band MIMO processing. In the non-coherent case, only one single lobe is present, and its dimension is determined by the range resolution of the radar, i.e. $1.5 \mathrm{~m}$ corresponding to the $100 \mathrm{MHz}$ bandwidth, and by the geometry of the system. As can be seen, the coherent MIMO processing reduces the dimension of the peak down to few centimetres, but, as mentioned above, it also introduces a great number of secondary lobes. From a processing point of view, the coherent MIMO elaboration is similar to a digital beamforming where a sparse antenna array is used. The sparse configuration of the antenna elements results in the presence of sidelobes, whose intensity and location depend on the geometry and the number of remote sensors, as well as on the carrier frequency [1]. Since the proposed radar network operates in two different frequency bands, the impact of the sidelobes can be thus strongly reduced. Merging together the two detections, the secondary lobes can't sum together due to their different position, thus reducing their resulting amplitude, while the main peaks at the two bands, whose location depends only on the actual target, sum constructively.

A dual-band MIMO radar system can reduce the undesired sidelobes without changing the system geometry and the sensor number (i.e. without hardware increasing). Furthermore, once calibrated, no additional processing for synchronizing and then fusing the multi-band acquisitions is requested due to the intrinsic coherence among the bands assured by the photonics-based signal generation and detection.

\section{CONCLUSION}

In this paper, a dual-band coherent MIMO radar network architecture based on photonic technologies has been presented, realized and characterized. Its performance confirmed the suitability of the system for coherent MIMO applications in the envisioned scenario, both detecting weak echoes and spatially resolving an emulated target. The superiority of such an approach compared with the non-coherent approach is apparent, thus demonstrating the validity of the proposed architecture. Photonic is an enabling technology for coherent MIMO radars with widely distributed antennas, thanks not only to its capability to flexibly generate and receive RF signals over multiple frequency bands assuring excellent coherence, but also to its capability to grant long-range signal distribution over fiber. Moreover, the WDM approach allows the system to resort to already existing optical networks, even sharing the fibers with telecom services, thus easing its deployment. The results presented in this paper should be considered as demonstration of the effectiveness of the realized prototype, prior to the already planned deployment of the system for the surveillance of a nearby harbour area. Further results are thus expected, aiding the development of future multi-sensor radar.

\section{REFERENCES}

[1] A. Haimovich, R. Blum, and L. Cimini, "MIMO radar with widely separated antennas," IEEE Signal Process. Mag., vol. 25, no. 1, pp. 116-129, Jan. 2008

[2] P. Ghelfi et al., "A fully photonics-based coherent radar system," Nature, vol. 507, no. 7492, pp. 341-345, Mar. 2014.

[3] F. Laghezza et al., "Field evaluation of a photonics-based radar system in a maritime environment compared to a reference commercial sensor," IET Radar, Sonar Navigat., vol. 9, no. 8, pp. 1040-1046, Oct. 2015.

[4] F. Scotti, D. Onori, and F. Laghezza, "Multi-band software-defined coherent radar based on a single photonic transceiver," IEEE Trans. Microw. Theory Techn., vol. 25, no. 11, pp. 757-759, 2015.

[5] J. Fu and S. Pan, "A fiber-distributed bistatic ultra-wideband radar based on optical time division multiplexing," in Proc. Int. Top. Meeting Microw. Photon. (MWP), Oct. 2015, pp. 1-4.

[6] F. Zhang, B. Gao, and S. Pan, "Photonics-based MIMO radar with highresolution and fast detection capability," Opt. Express, vol. 26, no. 13, pp. 17529-17540, 2018.

[7] S. Maresca et al., "Coherent MIMO radar network enabled by photonics with unprecedented resolution," Opt. Lett., vol. 45, no. 14, p. 3953, 2020, doi: 10.1364/OL.391296.

[8] I. Pasya and T. Kobayashi, "Detection performance of M-sequence-based MIMO radar systems considering phase jitter effects," in Proc. IEEE Int. Symp. Phased Array Syst. Technol., Oct. 2013, pp. 394-398. 